\title{
Modeling Incubation and Restructuring for Creative Problem Solving in Robots
}

\author{
Jerald D. Kralik ${ }^{1}$, Tao $\mathrm{Mao}^{2}$, Zhao Cheng ${ }^{2}$, and Laura E. Ray ${ }^{2,3}$ \\ ${ }^{1}$ Department of Bio and Brain Engineering, Korea Advanced Institute of Science and \\ Technology (KAIST), Daejeon, 34141, South Korea \\ ${ }^{2}$ Thayer School of Engineering, \\ Dartmouth College, Hanover, NH 03755 USA \\ ${ }^{3}$ Corresponding Author: 1ray@ dartmouth.edu
}

\begin{abstract}
Creativity represents the pinnacle of higher-level cognition, but exactly how it is achieved remains poorly understood, especially when simultaneously facing the opposing challenge of intractable complexity. The aims of the current study were (a) to examine how the brain may achieve the dual goals of creativity and complexity reduction, and (b) to begin developing higher-level cognition and creativity in robots. We addressed these aims by (a) modeling an example of insight problem solving and comparing it to empirical data, and (b) testing the model on a robot platform. Unlike other models, we propose a single mechanism for both creative problem solving and complexity reduction. Focusing on creativity, the computational mechanism leads to insightful problem solving by restructuring an internal belief representation based on evidence collected during an incubation period. Because insightful problem solving has been examined closely in nonhuman primates, providing detailed quantitative datasets lacking in humans, we tested the model by comparing simulations to insightful problem solving by rhesus monkeys. Results show that the proposed model accounts for both the discontinuous three stage problem-solving patterns and the spontaneous generalization to novel cases observed with the monkeys. To test the model in a physical environment, we implemented it in a vision-equipped robot, and the model solved the same insight problem from camera percepts. Our model shows how the creative brain may address the dual challenges of complex environments - finding unprecedented opportunity hidden within potentially intractable complexity - and suggests that both challenges may be met by a single underlying computational mechanism.
\end{abstract}

Keywords: robot creative problem solving; insight; computational cognitive neuroscience; evolution of higher-level cognition 


\section{Introduction}

Creative problem solving is a hallmark of higher intelligence, reaching its pinnacle in humans and leading to many of our greatest advances. Thus, an important avenue of research is to determine how the brain achieves it. A fundamental aspect of creativity is finding an unexpected solution to a challenging problem (Smith \& Ward, 2012; van Steenburgh et al., 2012). That is, most problems are solved by finding a solution path based on the most efficient, obvious or apparent possibilities. When such possibilities are not successful, nonapparent ones need to be considered. However, nonapparent possibilities are often difficult to discover, producing a sense of cognitive strain and remoteness during the discovery process. Why are such possibilities so difficult to uncover?

Complex environments have provided the evolutionary selection pressures for creativity; however, they also pose a daunting counter challenge: the 'curse of dimensionality'. There are simply too many potential states of the world, stimuli comprising the states, and actions to consider, making many problems intractable computationally. Thus, problems often need to be simplified, and research in cognitive psychology and behavioral economics has famously shown that we draw problem representations from the 'blooming, buzzing confusion' with heuristics that replace intractable problems with simpler ones that yield reasonable approximations (Kahneman, 2011; Kahneman, Slovic, \& Tversky, 1982). Generally speaking, problems can be simplified by representing only the most obviously relevant problem components. This strategy appears to be sufficient much of the time, but by definition, must also fall short. A processing system can potentially accept a certain level of failure, but the creative brain was not afforded this luxury to fail, and therefore evolved to reconcile these two opposing forces, enabling seemingly limitless consideration of possibilities, and yet also enforcing limits. Thus, one key 
aspect of the creative process is to move from the simpler, apparent problem components to a consideration of nonapparent possibilities (Smith \& Ward, 2012; van Steenburgh et al., 2012). But exactly how the creative brain does this remains unclear.

In this paper we propose a mechanism for this process. We take a parsimonious approach and consider the simplest underlying processes that may resolve these dual challenges. As with others, our modeling approach assumes that creative problem solving is ultimately composed of simple underlying associative processes, and can be modeled in a Markov Decision Process (MDP) and Reinforcement Learning (RL) framework (e.g., Sutton \& Barto, 1998). Within this framework we show how an algorithm we developed previously, the $Q$-Tree algorithm, can lead to both apparent and nonapparent problem solving. The Q-Tree algorithm was originally designed to cope with the computational complexity of realistic learning problems (Mao et al., 2012; Sun et al., 2011). It focuses on state representation and imagines that for most problems, many states that the agent might find him/herself in may require the same action to be taken to reach the goal state. If so, rather than treating every state uniquely, it significantly lowers the computational load if such states are treated identically (until proven otherwise). The algorithm uses $Q$ values (i.e., state-action pairing evaluations) and a hierarchical tree structure to represent the problem domain. Indeed, the algorithm improves learning convergence and reduces computational and memory resources by orders of magnitude relative to traditional learning models (Mao et al., 2012; Sun et al., 2011).

To show how the algorithm also achieves creative problem solving, we take a specific example and compare simulation results to empirical data. Because it is difficult to find unequivocal cases of creativity, insight problem solving proves useful since it has distinctive diagnostic features (see van Steenburgh et al., 2012). Insight normally involves an impasse being 
reached, in which a problem solution does not readily come to mind. Overcoming the impasse often requires an incubation period, followed by a restructuring of the problem representation that leads to a rapid solution. This impasse and subsequent incubation stage are important because they provide the strongest evidence that apparent possibilities have been tried and failed, and the problem solver is seeking a nonapparent solution. When such problems are eventually solved, they usually occur relatively suddenly with a sense of "A-ha!".

Even though insight problems are well documented in the literature, quantitative datasets detailed enough for computational modeling are lacking. Therefore, we leveraged studies conducted in comparative higher-level cognition, in which the same stages of impasse, incubation, and insight have been observed in nonhuman primates when solving insight problems, suggesting that the fundamental processes of creative cognition are evolutionarily conserved, at least among some primate species (Kohler, 1925; Passingham \& Wise, 2012; Striedter, 2005). More specifically, we modeled problem solving by rhesus monkeys on the reverse-reward problem, in which the subject is offered a choice between two food options, with one clearly better than the other (such as four food items versus only one), and they are given the option they do not choose; thus, they must avoid selecting the better option (apparent solution) and instead select the worse option to receive the better one (nonapparent solution) (Kralik, 2005; Kralik et al., 2002; Murray et al., 2005; Shifferman, 2009).

In addition to our first aim of examining fundamental processes underlying creative problem solving and their relationship to complexity reduction, the second aim of our study was to test the algorithm on a robot platform, and thereby (a) assess its ability to work in a more realistic, physical environment, and (b) help set the stage for creative problem solving in robots. To reach the second aim, we show that a robot can use the algorithm to solve the reverse-reward problem 
based on percepts derived from a camera input of the choice options, and can do so as effectively as or more effectively than a nonhuman primate.

In sum, the main contributions of the paper are twofold. One, to our knowledge this is the first proposal for how both creative problem solving and complexity reduction can be achieved via a single computational mechanism. Most other models of creative problem solving are qualitative, and thus require computational explication for further development. To this end, Hélie \& Sun (2010) developed a computational model of incubation and insight in creative problem solving, in which implicit and explicit processing modules interact to produce incubation and insight that match the behavior of human problem solvers in different high-level cognition and creative problem-solving tasks. However, it is not clear how such a model can account for the discontinuous learning curves found with creative problem solving that appear to reflect separable stages of explicit problem solving, restructuring (potentially multiple times), implicit incubation (i.e., problem solving with no apparent effect on behavioral performance), and insight. We extend the computational work in several ways, especially with respect to problem restructuring and learning curve discontinuities. Second, we test the model in a realistic physical environment, implementing it on a robot platform with visual inputs via a camera. We demonstrate reverse-reward problem solving by the robot, which provides further evidence for the validity and expandability of the algorithm. The robot implementation also helps set the stage for further development of high-level cognition and creative problem solving in autonomous systems.

In what follows, we describe the comparative cognition empirical studies we simulated; we present our computational model; we compare the simulation and experimental results for reverse-reward problem solving (including generalization to novel cases); we test reverse-reward 
problem solving by a robot; and finally, we discuss the implications of the findings.

\section{Description of reverse-reward problem tested with rhesus monkeys}

In Murray et al. (2005), six adult male rhesus monkeys (Macaca mulatta) were tested on a reverse-reward problem, in which when offered a choice between two quantities of food - one and four - they were given the quantity they did not choose (Fig. 1). Thus, to obtain the four food items, they needed to choose the single one. Twenty trials were conducted per session. The location (left or right) of the food amounts followed a pseudorandom order, with the constraint that for each block of 10 trials, five trials had the configuration $\{1,4\}$ (i.e., one on the left, four on the right) and the other five trials $\{4,1\}$. The monkeys were tested one session per day, five days per week. The criterion for successful performance was $90 \%$ correct responses (choosing the smaller food quantity to receive the larger) over five consecutive days (i.e., 90 correct choices or more in 100 trials).

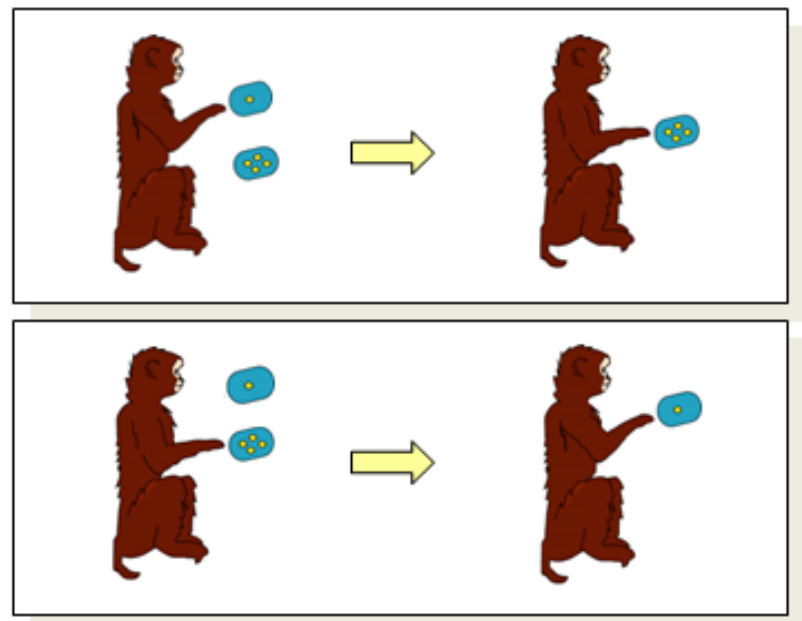

Figure 1. Illustration of the reverse-reward problem. The monkey receives the option it does not select.

This reversed-reward problem is difficult for nonhuman animals to solve, in part because it requires overcoming a strong, "prepotent" inclination to select the larger food quantity (Kralik, 2005; Kralik et al., 2002; Murray et al., 2005; Shifferman, 2009), as well as the ability to 
generate an innovative response of selecting the smaller over the larger in order to receive the larger quantity (Kralik, 2005). In fact, it appeared for some time that perhaps nonhuman animals could not solve the problem without it being simplified. Then Murray et al. (2005) found that rhesus monkeys could solve it on their own if given enough experience. Moreover, they discovered that the monkeys solved the problem in three distinct stages (Fig. 3a and c). In the first stage, they appeared to learn to inhibit the selection of the larger quantity; however, once this occurred, instead of selecting the smaller quantity, the monkeys entered what appeared to be an incubation stage in which their performance hovered at chance with no discernable signs of improvement. In the third stage, they began selecting the smaller quantity relatively rapidly, suggesting insight. Although the learning curves of individual monkeys spend different amounts of time in each stage, the characteristic three-stage learning dynamics are evident across all monkeys (see Fig. 3a and c).

Once the problem was solved an important follow-up question was, what exactly did the monkeys use to solve it? Did they learn simply to avoid the four peanut pieces and select the one piece or were they using something more abstract: e.g., to select the smaller quantity regardless of the quantity combinations? To determine the level of abstraction being used, a follow-up study was conducted with two additional rhesus monkeys (Kralik, 2011). Once the monkeys had solved the reverse-reward problem in the new experiment, reliably selecting one cereal pellet over four, with a performance criterion of $90 \%$ in the last 100 trials, transfer tests for novel quantities were conducted. In two 75-trial sessions, the following 15 combinations were tested: 1 vs. 2,1 vs. 3,1 vs. 4,1 vs. 6,2 vs. 3,2 vs. 4,2 vs. 5,2 vs. 6,2 vs. 7,3 vs. 4,3 vs. 5,3 vs. 6,3 vs. 7, 4 vs. 6 , and 4 vs. 7. Every combination was tested five times per session for a total of 150 trials per monkey. The study found that once the rhesus monkeys solved the problem with the 
single quantity combination of one versus four, they spontaneously generalized to other quantities. These strong transfer results are striking, especially when considering the cases when the quantity four was now the smaller of the two quantities. After hundreds of trials of learning to avoid the four food pellets, both monkeys nevertheless immediately selected the four-pellet option once it was paired with a larger quantity. These transfer results provide rare evidence that nonhuman animals sometimes spontaneously see the world in abstractions rather than merely as specific, concrete objects. Abstraction is of course a powerful cognitive tool to turn otherwise novel events into familiar ones (e.g., Gazzaniga et al., 2013; Holyoak \& Morrison, 2012; Russell \& Norvig, 2010).

While the reverse-reward problem may seem simple, it has merit in that (a) it has key features that require creativity in problem solving — in particular, having a potential problem solution that is readily apparent (selecting the better option) and one that is distinctly nonapparent to the monkeys (selecting the worse option to receive the better one) — and (b) its problem-solving profile reveals diagnostic features of insight: i.e., behavioral evidence for reaching an impasse, a period of incubation, then insight. In addition, it is the only such problem that we know of where detailed experimental data are provided for the entire process as well as for multiple individuals. As such, we believe that these rhesus monkey data sets offer valuable evidence to model fundamental features of creative problem solving. In the general discussion (Section 6.4) we discuss how the algorithm extends to other cases of creative problem solving, including those with human subjects.

In the following sections, we present the model, and show how it elicits the three stage problem-solving curve found in Murray et al. (2005), as well as the spontaneous generalization in Kralik (2011), suggesting that the model may capture fundamental processes underlying 
insight problem solving. We then perform the physical experiment in which a robot uses the model to build the requisite representation for solving the reverse-reward problem from camera percepts and subsequently solves the problem.

\section{Problem-solving model}

\subsection{Reinforcement Learning}

The model uses a Markov Decision Process (MDP) and Reinforcement Learning (RL) framework (e.g., Sutton \& Barto, 1998). A Markov Decision Process (MDP) is a mathematical model for describing temporal decision-making in stochastic domains. An MDP of a single-agent learning system is formally defined as a tuple $\langle S, A, P, R\rangle$, where $S$ is a set of states $S \in S, A$ is a set of actions $a \in A$ available to the learning agent; $P$ is a transition probability $P\left(s_{t+1} \mid s_{t}, a_{t}\right)$ that denotes the probability of transition from $s_{t}$ to state $s_{t+1}$ at time step $t+1$ when action $a_{t}$ is taken by the agent when in state $s_{t}$ at time step $t$, and $R$ is the reward function. For our modeling work, a state in an agent's hierarchical state representation is the internal state, denoted by $s$, the environmental state information observed by the agent is termed the environmental state, denoted by $e$, and the initial representation of the environmental state is the external state, denoted as $x$. An example of the state definitions for the reverse-reward problem is shown in Fig. $2 \mathrm{a}$, in which the environmental state consists of four food items on the left and one on the right (from the monkey's perspective); the external state is the quantity configuration $\{4,1\}$; and the hierarchical internal state $s$ is described in Section 3.2.

For reinforcement learning, Q-learning, a temporal-difference (TD) learning solution, is popular for solving MPDs because it requires modest memory and is model-free (Sutton \& Barto, 1998). In the actor-critic version of Q-learning (Crites and Barto, 1995), the critic module evaluates TD error, which is the difference between the current action value and subsequent 
reward, with the latter estimated as the sum of the actual reward received immediately after taking the action and an estimate of future rewards received by subsequent actions taken from the new state:

$$
\delta_{t}=\left[r_{t+1}+\gamma \max _{b} Q_{t}\left(s_{t+1}, b\right)\right]-Q_{t}\left(s_{t}, a_{t}\right)
$$

$r_{t+1}$ is the actual reward received at time step $t+1, s_{t}$ and $s_{t+1}$ are the states at time step $t$ and $t$ +1 , respectively, $a_{t}$ is the action taken at time step $t, b$ is the action with the maximum $Q$ value in $s_{t+1}$, and $\gamma \in(0,1)$ is the discount factor, which determines the value of rewards received in the future. For learning of the reverse-reward problem, each trial is considered to start with a state $s_{t}$ and end with the same absorbing state $s_{o}$ whose state value (i.e., estimated future reward) is zero, receiving a reward $r_{t+1}$ whose value is the quantity obtained - i.e., the opposite amount from what is selected (as described in Section 2).

The actor module updates the action value ( $Q$ value) in the following way:

$$
Q_{t+1}\left(s_{t}, a_{t}\right)=Q_{t}\left(s_{t}, a_{t}\right)+\alpha \delta_{\mathrm{t}}
$$

where $\alpha \in(0,1]$ is the learning rate. The actor module selects an action according to the $Q$ values associated with the current state. There are several methods for action selection, e.g., $\varepsilon-$ greedy, the softmax method, and a combination of both (Sutton and Barto, 1998). We used an $\varepsilon^{-}$ greedy-softmax method, in which the action with the largest $Q$ value is selected with probability $\varepsilon$, and otherwise an action is selected according to the Boltzmann distribution of $Q$ values:

$$
\pi\left(a \mid s_{t}\right)=\frac{e^{Q_{t}\left(s_{t}, a\right) / \tau}}{\sum_{b} e^{Q_{t}\left(s_{t}, b\right) / \tau}}
$$

where $\tau$ is the temperature that controls the degree of action exploration.

\subsection{Hierarchical state representation}

Conventional Q-learning discretizes every state a priori and $Q$ values for each state-action pair are updated individually throughout learning; thus Q-learning suffers the 'curse of 
dimensionality.' In contrast, Q-Tree learning is a variant of Q-learning derived in Mao et al. (2012) in which a hierarchical state representation is concurrently learned along with the action policy, thereby reducing state space complexity by considering only the minimum number of states necessary for learning. While a number of algorithms exist for learning hierarchical state representations (e.g., Uther and Veloso, 1998; Pyeatt and Howe, 2001), Q-Tree learning works on both continuous and discrete state spaces, requires no a priori discretization of the state space if it is continuous, has computational complexity that is linear with state dimension, and also can eliminate state dimensions that are found to be irrelevant to the problem (Mao et al., 2012).

The intuition of the algorithm is illustrated in Fig. 2 for the reverse-reward problem. On a given trial, the environmental state is the choice offered to the monkey: one food item to the monkey's left, four to the right. Thus, as shown in Fig. 2a, the external state space can be represented by a two-dimensional plot of the quantities presented. Any given external state $x$ ("+" mark in Fig. 2a), then, is processed to determine the internal state $s$. In Fig. 2a, it is assumed that the monkey begins the experiment with a "prepotent" internal state representation. At the top of the hierarchy is a super state, comprised of all external states experienced by the monkey. After sufficient experience, the super state can be divided into sibling states (described below which has already occurred in this prepotent representation). When this occurs, the parent node (i.e., the super state) is considered the separation node, and the siblings the internal state nodes. For the reverse-reward problem with the choice options based on quantities, the internal representation divides the external state space into two regions separated by the diagonal, in which the left state node covers all cases in which the right quantity is the larger option, and the right state node all cases in which the left quantity is the larger option. Thus, when the monkey experiences the external state "+”, it is processed through the hierarchy from the super state 
along a judgment path (in this case, the one step indicated by the arrow in Fig. 2a). The monkey then chooses a response option (left or right) based on (a) the $Q$ values for each response option associated with the right internal state node in the hierarchical state representation and (b) the action selection mechanism described in the previous section.

The hierarchical representation is constructed upon a decision-tree structure using a topdown scheme: again, the state-space representation begins by clustering all visited external states into one super state, and the internal state space is then sequentially separated into subspaces when evidence exists for separation as learning proceeds. Separation can be driven by the similarity of any number of external state features: for the reverse-reward problem, it is based on received rewards. Internal state pruning can also occur when evidence exists for rejoining the sibling states, as illustrated in Fig. $2 \mathrm{~b}$ (depicting only the internal state nodes at three time periods). Here, the initial or prepotent internal state representation is depicted by having two separate internal states representing the quantity configurations $\{1,4\}$ and $\{4,1\}$ at the top of the figure. At that time, the monkey would be treating each configuration differently: for example, reaching to the right for $\{1,4\}$ and to the left for $\{4,1\}$. However, the two internal states may be joined into a single internal state when experiential evidence accumulates to indicate incorrect internal beliefs (e.g., when selecting the larger reward leads to receiving something less than expected). Subsequent restructuring of the internal state space may then occur via separation as learning continues. The joining and separation of the internal states thus represents instances of problem restructuring. Incubation periods occur as the algorithm accumulates data that determines whether subsequent restructuring is warranted. For the reverse-reward problem, the restructuring of the initial prepotent state representation gives rise to incubation and subsequent restructuring dynamics during problem solving, which we examine in Section 4. 
(a)

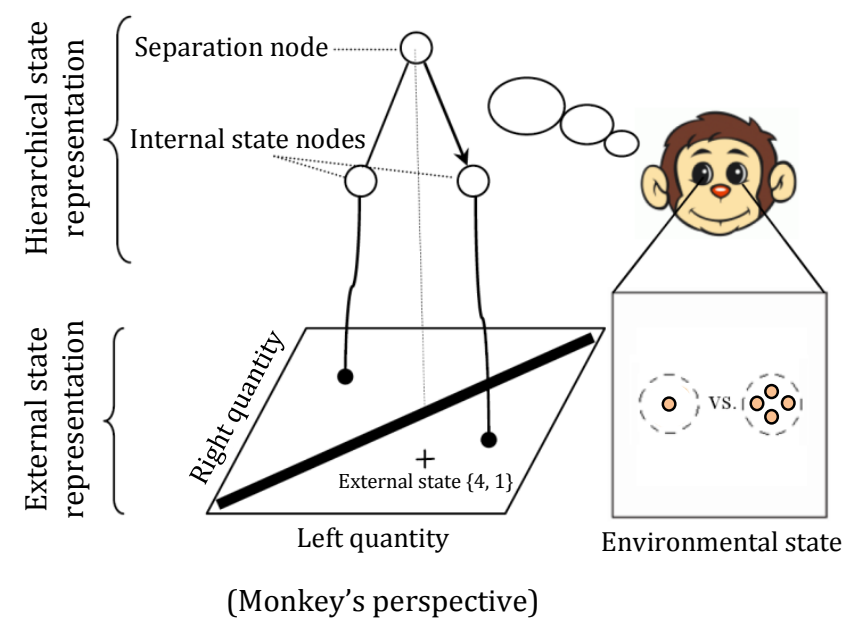

(b)

(1)

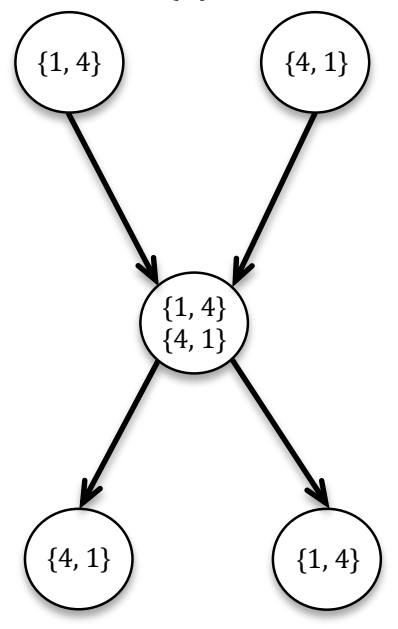

Figure 2. (a) Illustration of hierarchical state representation, (b) Illustration of internal states at three time periods. Restructuring occurs through pruning $(1 \rightarrow 2)$ and splitting $(2 \rightarrow 3)$.

Regarding separability, intuitively, internal states should be separated if they are composed of external states that require different actions to reach the goal. At the same time, to avoid

Table 1 summarizes the Q-Tree algorithm. After making a decision (algorithm step 4), taking action (step 5), and then updating based on the action's outcome (steps 6-8), data collection occurs. In the data collection phase for the reverse-reward problem, the algorithm gathers at each time step the data tuple $\left\langle x_{t}, r_{t+1}\right\rangle$ associated with the internal belief state-action pair $(s, a)$ (step 9), and counts the number \#(s,a) of data tuples collected for such a pair (e.g., for external state $\{1,4\}$, the number of trials in which the right action, selecting the quantity 4 , was taken). When all the numbers \# $(s, \cdot)$ for a pair of sibling states - for the reverse-reward problem, the number of trials for both left and right actions taken for both external states $\{1,4\}$ and $\{4,1\}$ - exceed half the sampling window $\omega$, the algorithm assesses internal state joinability (step 10). Similarly, when all the numbers \#(s,') for an internal state $s$ exceeds $\omega$, the algorithm assesses internal state separability (step 14). In the phase of assessing joinability, the algorithm evaluates sibling state similarity by calculating the cosine distance of vectors of the $Q$ values of the sibling states. If the similarity shows evidence of rejoining the sibling states, internal state pruning occurs. 
Regarding separability, intuitively, internal states should be separated if they are composed of external states that require different actions to reach the goal. At the same time, to avoid

Table 1. Q-Tree algorithm: Q-learning based on hierarchical state representation

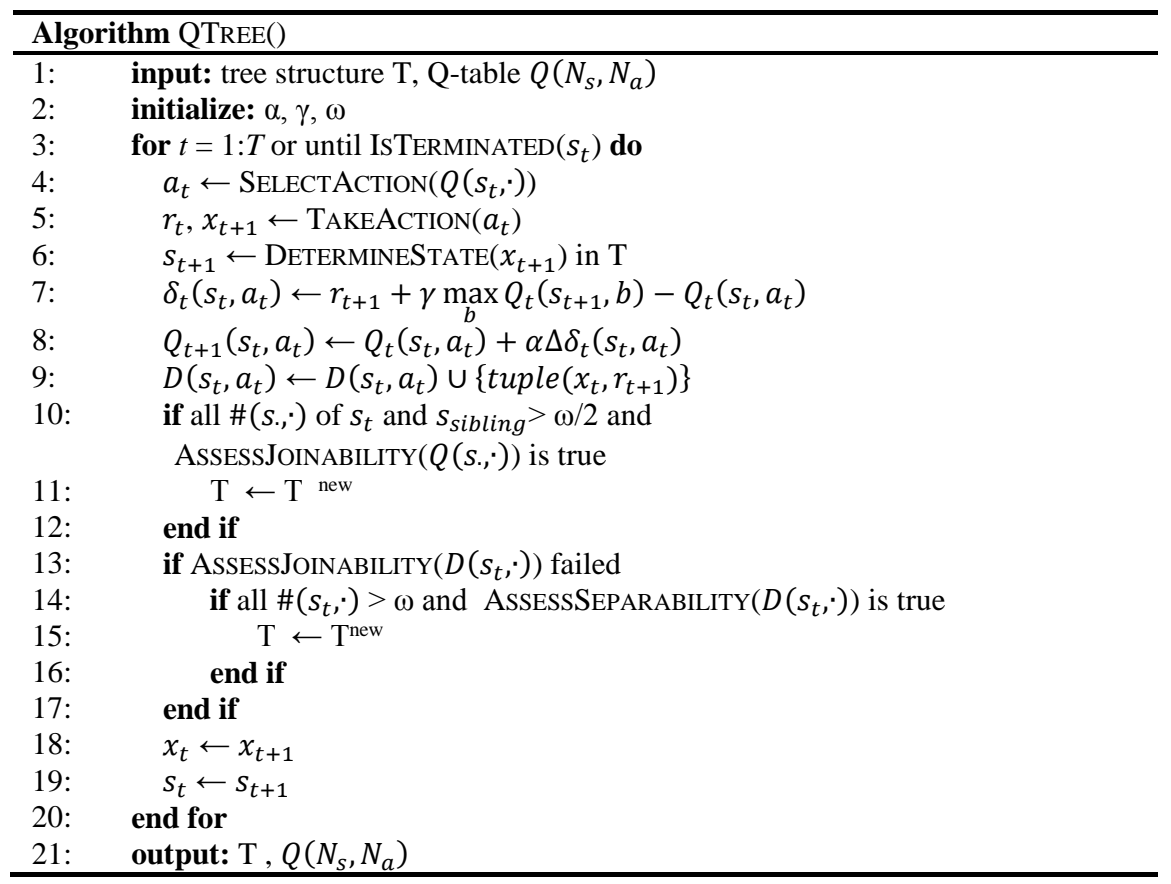

overly complex internal representations, external states should be separated (i.e., organized into different internal states) if the separation makes sense in external state space - i.e., if they are also separable in external state space. This latter condition is trivial for two external states (such as in the reverse-reward problem), but becomes important with problems involving larger external state spaces that require more sampling during learning (Mao et al., 2012; Sun et al., 2011). Thus, separability involves establishing these two conditions of being separable in both (1) $r$ (or $Q$ ) space and (2) external state space (Mao et al., 2012).

More specifically, we summarize the six steps in the Q-Tree algorithm for assessing internal state $s_{t}$ separability and, when needed, constructing an internal state boundary (details are described in Mao et al., 2012). First, for each action, one-dimensional clustering is performed based on the dimension of interest. For multistep problems this dimension is the change of $Q$ 
values, but for the one step reverse-reward problem (i.e., choice response receives reward), we use the $r$ values. The $r$-value data points are then labeled according to these clusters. Second, the $r$ values are then mapped to their external states, and the external states are grouped according to the cluster assignments. Third, now focusing on the external state space, a linear classifier is found based on the grouped external state data (trivial for the reverse-reward problem). Fourth, the external state data are separated in external state space by the linear classifier and mapped back to the $r$ values. Fifth, the linear classification is assessed statistically using the $r$ values to determine whether the separation should be conducted. Sixth, the internal state is separated only if the statistical evidence supports it.

As stated above, the joinability and separability components of the algorithm are our proposed processes for problem-solving restructuring, while data history collection is that for incubation. In addition, the overall coordination of these processes — our Q-Tree algorithm in general — represents our model of cognitive control, involving both implicit processing components, i.e., those conducted "in the background" (e.g., data history collection), and explicit ones (e.g., working memory and decision making on each trial) (e.g., Dijksterhuis et al., 2006; Gazzaniga et al., 2013; Miller \& Cohen, 2001).

Finally, to model the experimental findings for the reverse-reward problem, two assumptions were made to deal with uncertainty in real experiments. First, in the action policy selection, the model introduces a confusion parameter to represent the agent's uncertainty during learning:

$$
C(\mathrm{~s})=\frac{\min (Q(s, \cdot))}{\max (Q(s, \cdot))}
$$

Confusion increases when the minimum and maximum $Q$ values are similar, and the agent may lose interest in exploring actions. This behavior of reducing exploration in the face of uncertainty relates to action biases often seen in animal learning (such as selecting the left option every trial), 
and may in fact reflect a clever strategy to simplify the problem space when confusion is high (which we discuss further in Section 6.1). At the same time, different agents exhibit different behavioral patterns in the face of uncertainty in learning, and we modeled this as greediness $\varepsilon$ for action selection parameterized by $\kappa$ :

$$
\varepsilon=C^{\kappa}
$$

where $\kappa$ denotes the degree to which confusion affects action selection, such that if $\kappa$ is high, for example, confusion will result in more deterministic behavior.

Second, due to evolutionary pressure, animals, including humans, have developed number sensing systems with size and distance effects, i.e., number perception varies for different external stimuli and depends on the numerical value of the stimulus (Dehaene, 1998, Feigenson et al., 2004). We adopted the linear variability model (Weber-Fechner's law) (Feigenson et al., 2004) to model number perception in the brain, where the number variation follows a normal distribution with a variation proportional to the number size: i.e., for number $N$, number perception is considered to be sampled from the normal distribution with a variation of $k N$.

\section{Comparison of simulations to experimental data}

In this section, we use the Q-Tree model to simulate solving of the reverse-reward problem, and we compare the simulation results to the experimental data from the rhesus monkey studies described in Section 2. We show that the individual behavioral dynamics observed in rhesus monkeys can be modeled by tuning model parameters $\alpha$ (learning rate), $\tau$ (exploring temperature), $\omega$ (sampling window), and $\kappa$ (confusion effect). We then test the computational model's ability to explain generalization to novel quantities.

Similar to the experimental procedure described in Section 2, in simulation experiments, each learning block consisted of 20 trials that presented the problem-solving agent with either ' 1 vs. 4' 
or ' 4 vs. 1'. The two cases were balanced within the first and second half of the learning block as occurred in the original experiment in Murray et al. (2005). Initially, the problem-solving agent has an existing state representation that distinguishes ' 1 vs. 4 ' and ' 4 vs. 1 ', and has a prepotent response of selecting the larger quantity. This response is modeled by setting the $Q$ value associated with selecting the larger quantity to provide a high probability of selecting the prepotent response. The criterion for completing the learning was $90 \%$ correct responses in five consecutive learning blocks, as in the main experiment in Section 2. Table 2 shows the model parameters used for each problem-solving agent.

Table 2. Model parameters for agents solving the reverse-reward problem

\begin{tabular}{lllll}
\hline & $\alpha$-learning rate & $\tau$-exploring temperature & $\omega$-sampling window & $\kappa$-confusion effect \\
\hline Agent 1 & 0.01 & 1 & 60 & 3 \\
Agent 2 & 0.0048 & 1.1 & 20 & 3 \\
Agent 3 & 0.012 & 1 & 20 & 3 \\
Agent 4 & 0.012 & 2.1 & 120 & 1.4 \\
Agent 5 & 0.012 & 1.3 & 95 & 3 \\
Agent 6 & 0.015 & 0.8 & 5 & 5 \\
\hline
\end{tabular}

\subsection{Solving the reverse-reward problem}

Figure $3 \mathrm{a}$ and $3 \mathrm{~b}$ show learning curves of the six rhesus monkeys and six simulated agents, respectively, and Fig. 3c compares the average learning curve from the experiment and simulations. In general, the learning curves consist of three distinct stages (Fig. 3a, 3b, 3c): in the first stage, the success rate quickly reaches approximately chance level (50\%); the second stage remains for a visible duration, fluctuating within roughly $40 \% \sim 60 \%$; in the final stage, the problem-solving agents achieve the criterion level (90\%) rapidly. In simulation, the halting of performance improvement after the first stage is due to internal state pruning (filled circles in Fig. 3b). Although the agent obtains a reward when selecting the larger quantity, it is less than expected (one instead of four), leading to a reduction in the original $Q$ values associated with selecting the larger quantity (i.e., left with internal state $\{4,1\}$, right with $\{1,4\}$ ). However, 
rather than selecting the smaller quantity as its $Q$ values rise, the agents become increasingly confused and also reach the point at which the two internal states appear identical (with respect to $Q$ values), causing a recombination of the internal states (pruning), and thus problem restructuring. Note that this first restructuring does not lead to the appropriate problem solution in that the two external states $\{1,4\}$ and $\{4,1\}$ are treated identically.

Evidence for internal state pruning is seen in the actual monkey behavior. At the transition between the first and second stages, all of the monkeys developed position biases regardless of the presentation configuration (i.e., $\{1,4\}$ or $\{4,1\}$ ). Thus, both external states were being treated identically, i.e., as if the monkey was experiencing the same state, and something like "when the food items are presented" became the one internal state controlling behavior. In the ensuing incubation period, the monkeys obtain evidence regarding the appropriate internal state representation; even though their current action goal is to select the left (or right) option every trial regardless of the quantity configuration, resulting in no outward sign of improvement, they begin to notice at some level that different outcomes occur for the different quantity configurations. The critical point is that even though the specific quantity configurations per se are not controlling behavior, i.e., having no effect on performance, they are nevertheless having some accumulative effect, which we designate as being "in the background", and thus a type of incubation. Once this acquired experience reaches threshold, the quantities themselves begin to control behavior again, leading to the rapid learning in the final stage. In the model, once the evidence of an appropriate internal state representation emerges it leads to a re-splitting of the one internal state into two (open circles in Fig. 3b), producing a second restructuring that now is correct, in which the action value is updated correctly and the performance increases rapidly.

Differences in behaviors across individual monkeys (and simulated agents) stem from 
different internal models of the learning agents, which can be interpreted as reflecting distinct

(a)

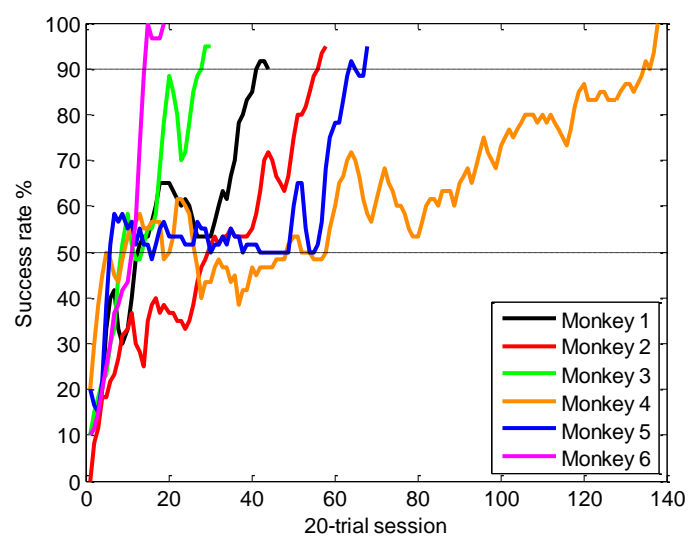

(c)

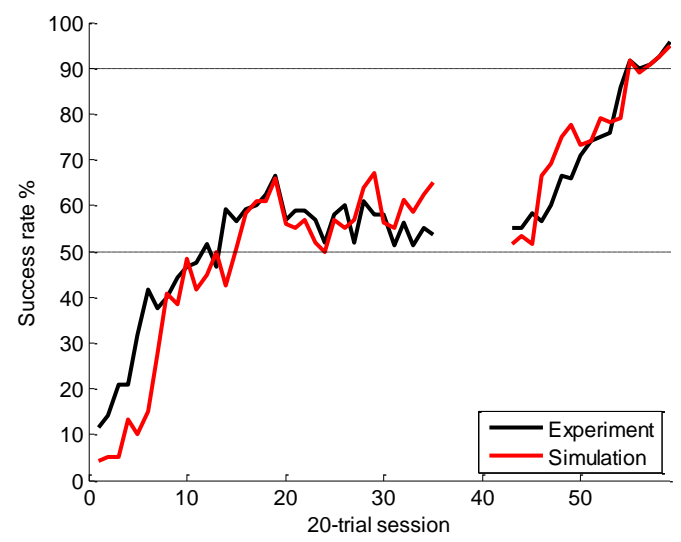

(b)

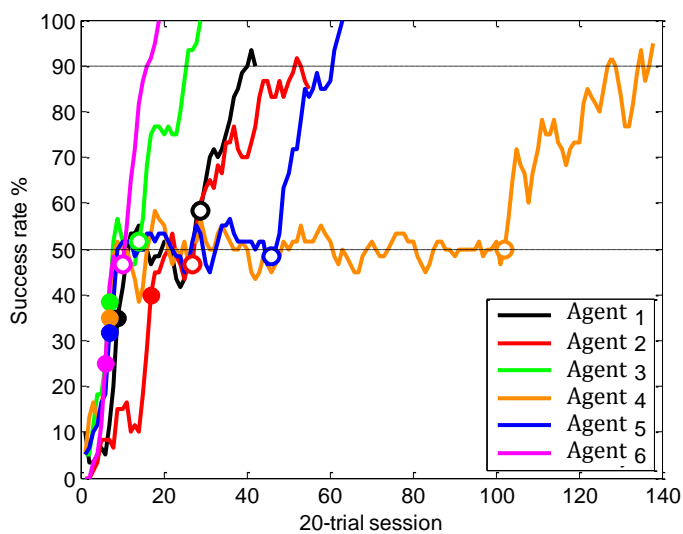

(d)

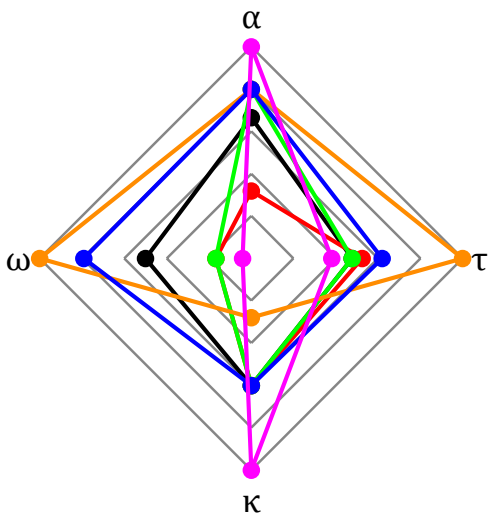

Figure 3. (a) learning curves for rhesus monkeys in the experiment; (b) learning curves for learning agents in simulation; (c) group mean learning curves for experiment and simulation; (d) starplot shows normalized model parameters of simulated agents, indicating different personalities of the monkeys exhibited in the experiment.

"personalities" of the monkeys (Fig. 3d). Indeed, it has been found that, like people, nonhuman primates tend to have stable cognitive traits, such as learning capabilities (e.g., Banerjee, 2009). From our modeling, Agent 6, representing Monkey 6, is the quickest learner (largest $\alpha$ ) and strongest empiricist (smallest $\omega$ ); Monkey 4 is the most adventurous (largest $\tau$ ), while it is also the laziest (smallest $\kappa$ ), and the weakest empiricist (largest $\omega$ ); Agent 1, representing Monkey 1, is an "average" monkey whose model parameters are average in value. 
The point of showing that individual behaviors can be modeled is that it is important for any proposed computational model of cognitive behavior to be able to model the rich variation in behaviors observed — the individual differences — and not just the general, average behavior, as is often done.

\subsection{Transferring experience to new quantity combinations}

We next focus on how the algorithm transforms stimulus inputs into higher levels of abstraction (e.g., Holyoak \& Morrison, 2012; Russell \& Norvig, 2010). That is, rather than treating the food item inputs as two specific "hand-coded" external states, the algorithm can treat them as quantities more generally, and thus generalize beyond the specific training exemplars to novel states made up of new quantities and quantity configurations.

Since Agent 1 has average model parameters, we used its parameter set to simulate transfer of the learned policy to novel combinations of numbers of food items; and we compared the results with those from the two monkeys in the second study described in Section 2 on quantity transfer. Figure 4 shows the actual results of transfer learning for the two monkeys (Monkey 1 and 2) and the simulation results for the learning parameters for Agent 1 of the prior simulation. For each experimental or simulation agent, the bars from left to right represent the success rate of case categories of (a) the last training session (100 trials), (b) all trials in the transfer sessions, (c) novel quantities only, and (d) trials with the quantity four being the smaller quantity. Note that transfer test (d) is the most difficult case, given that the agents solved the original problem by learning to avoid the quantity 4 , and yet must now immediately select it when paired with a larger amount.

Because the boundary constructed for the state representation during learning is approximately the diagonal in the external state space representation in Fig. 2a (thick line), the 
agent can consider other quantity combinations by determining whether the combination lies

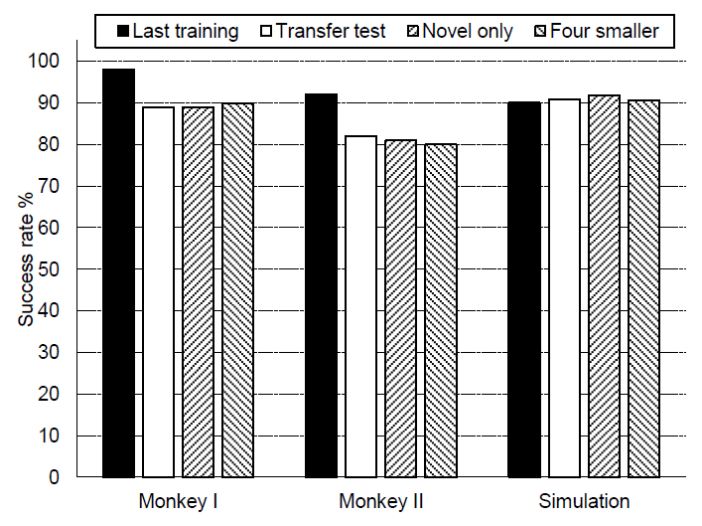

Figure 4. Comparison of experimental performance on transfer test with results from Q-Tree learning to the left or right of the boundary. Thus, the agent can transfer its knowledge and action policy from the previous problem to novel ones and solve them properly (Taylor and Stone, 2009).

In both the empirical data and simulation, however, the solution of the reverse-reward problem was transferred to different degrees depending on the actual quantity combinations. We hypothesized that the actual quantities of food presented in the transfer trials and the number perception systems of the brain may themselves affect transfer performance. Figure 5 represents the quantity effects on transfer learning for the two monkeys in the transfer study and the simulated monkey, showing the error rate (instead of success rate) by the relative size of the circle for each numeric combination of food items tested. The simulation results generally exhibit consistency with the experimental results, with the transfer test error rate in simulation being either similar to or bounded by the experimentally observed values. The experiment and simulation results reveal distance and size effects: as quantity distance between the choice options increases (e.g., distance 1 to 2 for ' 1 vs. 2' and ' 1 vs. 3 '), transfer performance improves (thus, generally smaller error rates represented by smaller circles along the direction off the diagonal in Fig. 5, i.e., black horizontal arrow); if the quantity distance remains the same, transfer performance worsens as the quantity size increases (e.g., ' 1 vs. 4' and '2 vs. 5') (thus, 
higher error rates represented by larger circles along the diagonal direction in Fig. 5, i.e., blue arrow). The similarity of experiment and simulation errors supports our hypothesis that some transfer errors were likely due to the nature of noisy number perception in the brain rather than failure of transfer to novel cases.

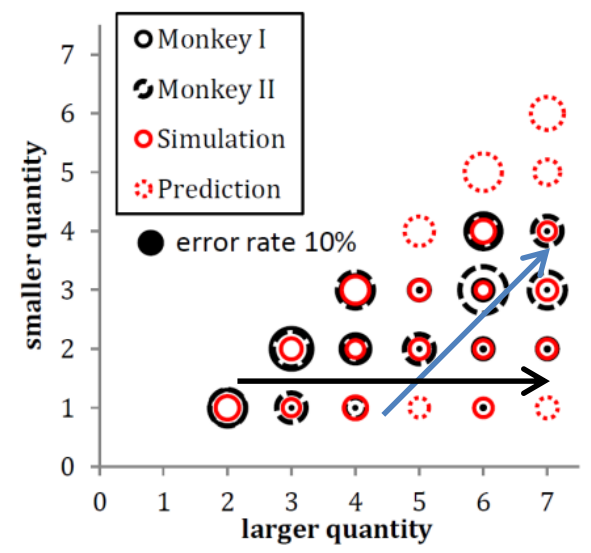

Figure 5. Quantity effects on transfer performance of different quantity combinations. See text for details.

\section{Reverse-reward problem solving by a robot}

In addition to our first aim of understanding the processes underlying creative problem solving and complexity reduction, the second aim of our study was to test the algorithm on a robot platform to verify its ability to work in a more realistic, physical environment, and to help in the development of creative problem solving in robots. Thus, we next tested whether a robot could use the algorithm to solve a version of the reverse-reward problem.

We used a Mobile Robots Pioneer P3-AT platform equipped with a CCD camera vision system. The experiment differed from the simulation experiments in that the agent must generate its internal state representation from visual percepts, rather than hand-coded states. To conduct the reverse-reward problem with a robot, we used a second computer system to present the choice to the robot by displaying the two options on a computer screen simultaneously, with one option being a single grape and the other four grapes. The robot's internal state representation 
was then formed from features extracted from an image of the display taken by the robot's camera (see Fig. 6a). A simple feature extraction algorithm counted the number of pixels with similar color to represent the quantity of grapes in each cluster in the image. The internal action delivered by the algorithm (choose the smaller or choose the larger quantity) was then translated and executed by the P3-AT robot platform. The external action set consisted of two types of camera movement: pan heading and tilt heading. Pan heading, either to the left or to the right, indicated the action of choosing the quantity on the left or on the right. Similarly, the tilt heading, either up or down, was the robot's means of acknowledging the reward received, either the larger quantity (up) or the smaller quantity (down).

Each experimental trial consisted of five stages as illustrated in Figure 6. First, the robot's camera took an image of the computer screen and processed the image to determine the internal state. Second, the robot selected an action based on the internal state derived from the image. The robot represented this action by panning the camera. Third, after a one second delay, the camera returned to its default position of facing forward. Fourth, the reward was given by removing one quantity from the screen; the quantity remaining on the screen indicated the actual reward. Finally, the robot perceived the reward by determining which cluster remained. The robot acknowledged the reward it perceived by changing the camera's tilt angle.

The problem-solving model was initialized with a representation having the prepotent knowledge, i.e., a large $Q$ value associated with choosing the larger amount. Unlike the simulation experiments, for the robot experiment we selected Q-Tree model parameters that resulted in more rapid learning than that observed in the rhesus monkeys: $\alpha=0.5, \tau=0.5, \omega=2$, and $\varepsilon=0.3$, corresponding to an effective value of $\kappa=1$ for the steady-state maximum and 
minimum $Q$ values within the experiment. The success rate and number of internal states are shown in Figure 7. From trials 1 to 4, the number of internal states was 2, and all actions selected

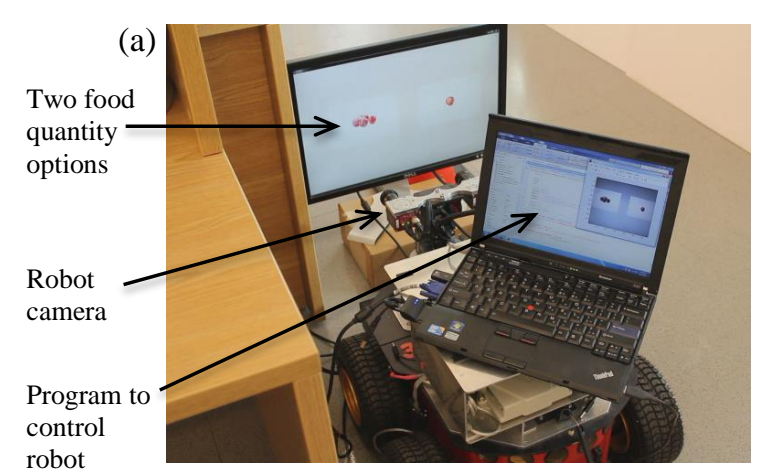

(b)
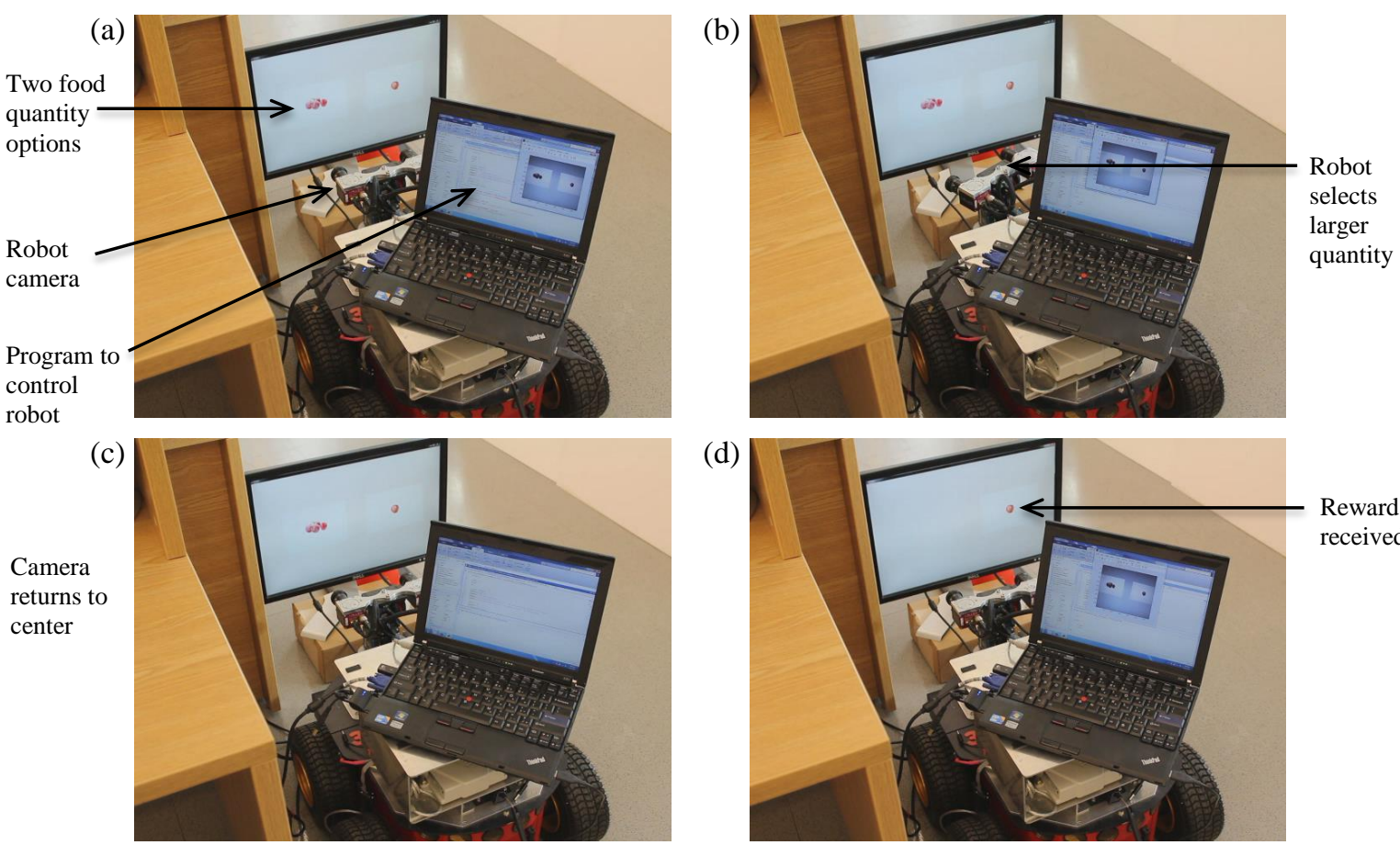

(d)
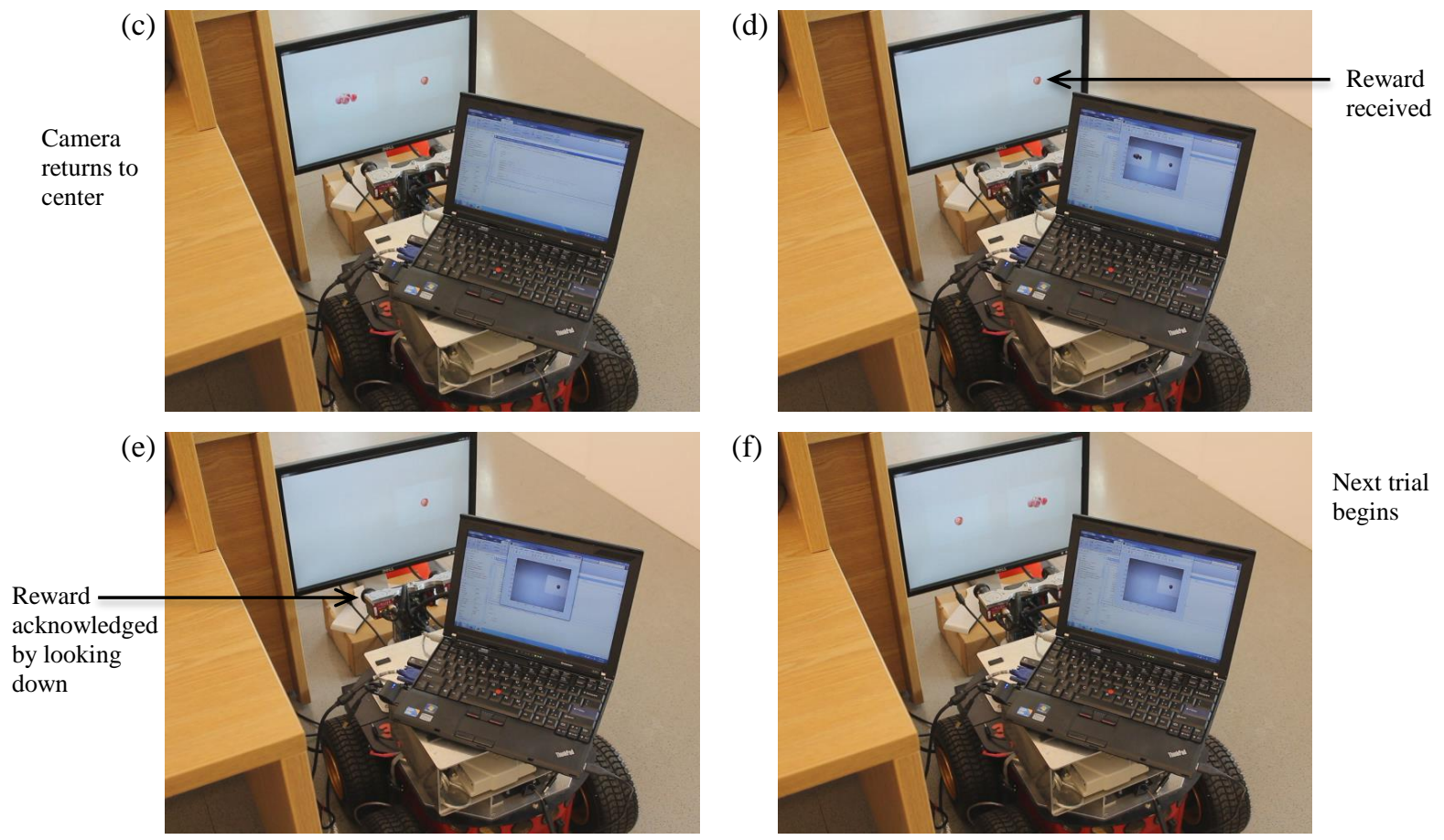

(f)

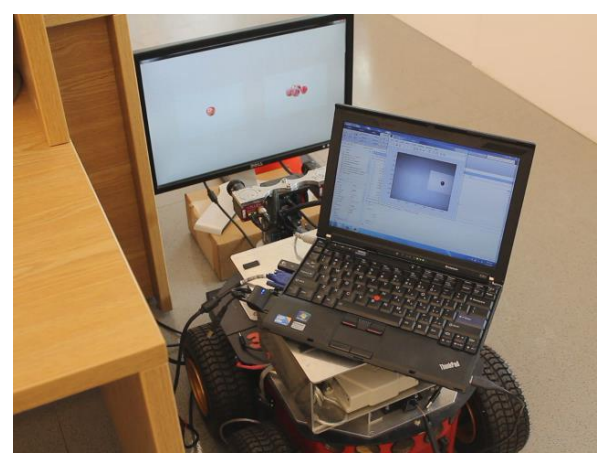

Next trial begins

Figure 6. Stages of the reverse-reward learning trial: (a) robot views a computer screen containing an image of two quantity options; (b) robot selects an option by looking at it; (c) robot looks straight ahead while waiting for a reward; (d) the quantity not received is removed from the screen; (e) the robot indicates that the smaller reward was perceived by tilting its head down; (f) a new trial begins.

by the robot were to choose the larger quantity, resulting in a $0 \%$ success rate. The actions were incorrect because the action selection was strongly biased by the prepotent representation. At trial 6 , the number of internal states decreased to one indicating the pruning of the tree and thus 
joining of the two internal states after evidence of incorrect action selection had sufficiently accumulated. From trials 6 to 27, the success rate of the algorithm averaged $\sim 50 \%$ because the two internal states were joined, and thus the action selection was effectively random. At trial 28, the model had collected adequate evidence for separation of the internal state and found the correct state representation for the problem. The three phases of problem solving are evidenced by the initial phase of rapid progression towards tree pruning (trials 1-5), incubation (trials 6-27); and insight (trials 27+).

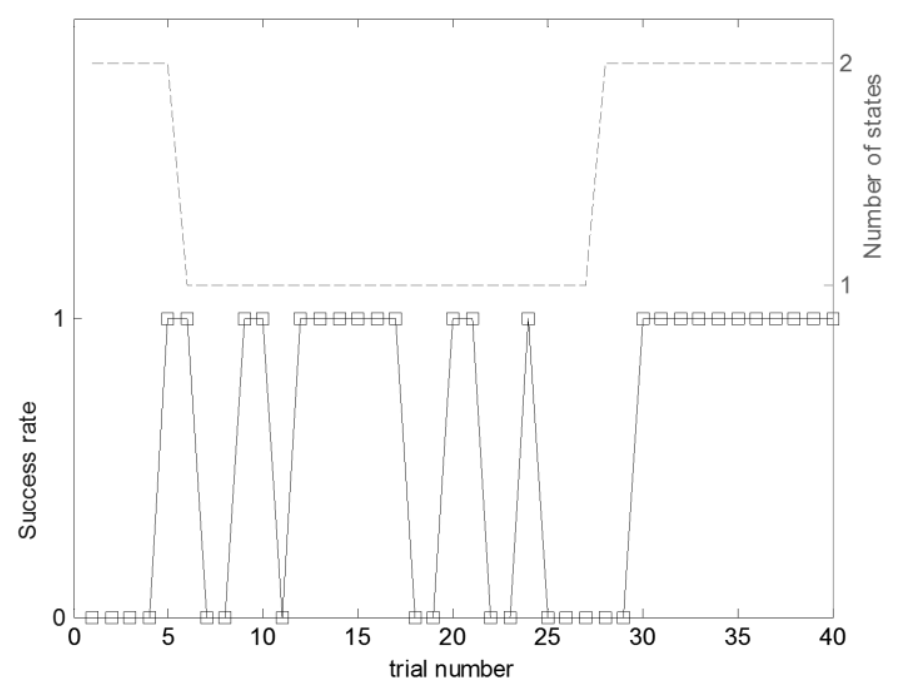

Figure 7. Results of the reverse-reward robot experiment

In situations where time, errors, and other cost factors are magnified, such as in robotics, it is of interest to empirically identify sets of parameters that result in the most rapid learning. We identified model parameters that provided convergence of the new representation within 27 trials, compared to a minimum of $\sim 400$ trials observed in the rhesus monkey experiment (Monkey 6). Of note, model parameters that elicited the learning dynamics for Monkey 6 in Table 2 were $\alpha=0.015, \tau=0.8, \omega=5$, and $\kappa=5$, while for rapid convergence in the robot experiment, we set a substantially larger learning rate of $\alpha=0.5$, with $\tau=0.5, \omega=2$, and $\kappa=1$. 


\section{Discussion}

To understand how creative problem solving in complex environments is achieved by the human brain (and other species who share the fundamental features of the ability), we examined how our Q-Tree algorithm would solve an insight problem and compared the results to empirical data. Because rich datasets are lacking for human creative problem solving, we turned to comparative high-level cognition research, in which insight problem-solving phenomena — impasse, incubation, restructuring, and rapid problem solving — have been observed, suggesting that this cognitive ability is conserved evolutionarily, at least among some primate species. Indeed, evolutionary neuroscientists have hypothesized that creative problem solving may have originated with the evolution of what is called granular prefrontal cortex (including lateral PFC and frontal pole) in primates (Passingham \& Wise, 2012; Striedter, 2005). In the reverse-reward problem, in which a better and worse option are presented and the subject must learn to select the worse option to receive the better one, most nonhuman animals appear to fail, typically perseverating at chance level performance. However, when given enough time, rhesus monkeys break through this perseverative stage and spontaneously solve the problem. Our model also passes through these stages. In fact, the close matching of simulation and empirical results lends support for our model as a possible mechanism underlying such problem solving. Hierarchical

state representation and our Q-Tree algorithm propose specific processes (joining and separating of internal states) for a way of restructuring the problem representation. During the incubation stage, the brain accumulates evidence for problem solutions, and the resulting internal state representation restructuring can trigger the appearance of spontaneous insight in problem solving, and lead to successful transfer to novel experiences.

\subsection{Computational modeling insights}


Our computational model and findings provide multiple insights into higher-level cognition and creative problem solving. First, because the algorithm was originally developed to help explain how higher functioning biological organisms manage the complexity of real-world environments, our model shows how a single mechanism may underlie the management of the two opposing processes - one that lowers complexity, and the other that utilizes the complexity to find creative solutions. Indeed, the need to have a mechanism that reduces complexity, yet at the same time provides a means for progressive access to increasing complexity might itself be a source of what it means to be creative. That is, because the brain appears to organize states, stimuli and actions into apparent and nonapparent categories, this organization may lead the nonapparent, less accessible solutions to appear creative. In any case, our model shows how potentially disparate phenomena (e.g., complexity reduction and creativity) may nonetheless result from the same relatively simple underlying mechanism(s).

Second, our model provides an example of the significance of hierarchical abstraction for the organization of higher-level information in the brain. Hierarchical structures enable dimension reduction at higher levels of abstraction, while at the same time retaining the ability to consider more options in the hierarchy when necessary (e.g., Fuster, 2008; Holyoak \& Morrison, 2012; Russell \& Norvig, 2010; Sun et al., 2011). This structure provides savings in other ways, including affording reduced sampling of the external state space when facing novel problems.

Third, our model provides impetus for the view that perception and cognition do not occur in a vacuum; rather, they are carried out by animals to determine what to do: to act. In our model, environmental states are processed (in the hierarchical representation) according to how they relate to goal attainment. Thus, it is too limiting at best, and potentially misguided at worse, to consider perception, cognition, and action separately; they evolved together and thus are perhaps 
best understood from the perspective of complete behavioral acts, with all components of the perception-cognition-action circuit actively involved in goal attainment (and thus including significant feedback/recurrent connections in the underling neural circuitry, as well as significant interactions with other systems) (Gazzaniga et al., 2013; Pfeifer et al., 2007).

Fourth, the Q-Tree algorithm provides a specific example of the tight interplay between learning and reasoning processes in high-level cognition. For example, reasoning processes determine whether internal states should be combined or split, while learning provides the 'data' necessary to make these determinations. Moreover, the reasoning processes may also 'see' the learning data at different levels of abstraction, providing seamless generalization to novel experiences (e.g., Holyoak \& Morrison, 2012; Russell \& Norvig, 2010). For instance, in our reverse-reward example, the experienced environmental states of one and four food items were represented as quantity configurations $(\{1,4\},\{4,1\})$, which in turn were treated as representative exemplars from a larger, more abstract representation of possible quantity configurations (the external state space representation in Fig. 2a). This abstraction ability allowed the agent to transfer learning from the original quantity configurations comprised of 1 and 4 to all others subsequently experienced in the transfer trials. This is another example of the significance of abstraction in higher-level cognition, with the inputs to the hierarchical state space representation (i.e., the external states) themselves potentially being represented at different levels of abstraction.

Fifth, an important feature of the algorithm is data collection 'in the background', in the sense that even though a given stimulus (such as the smaller quantity) is not controlling behavior, the individual is nonetheless learning something about it, which will eventually and rather abruptly come to control behavior. This modeling of the empirical data thus suggests that 
problem solving can occur inadvertently, when the agent is not attending to the potential problem solution. Although there is strong evidence that significant higher-level cognitive processing in humans occurs subconsciously (e.g., Dijksterhuis et al., 2006), we believe this modeling result demonstrates a clear implicit-explicit distinction in nonhuman animal problem solving for the first time; and our model provides a rare computational account of implicit versus explicit processing, and how they are coordinated via cognitive control (Gazzaniga et al., 2013; Hélie \& Sun, 2010; Miller \& Cohen, 2001).

Sixth, the parameterization of the model necessary to account for the rich variation in behaviors across individuals highlights the significance of individual differences and internal factors in higher-level cognition, such as in learning speed, length of incubation stage, and concentration on the task (Fig. 3d star plot). Our model thus shows how individual personality traits can be parameterized, which enables more rigorous examination of the relationship of specific internal factors with problem solving and creativity.

Finally, with respect to specific internal parameters, the model provides particular insight into confusion. The confusion concept in the computational model can drive agents into action biases (e.g., selecting the same option location, such as left). Although action biases are generally considered a sign of confusion and capitulation by subjects in behavioral studies, our model highlights how these biases can reflect an underlying cognitive strategy — one that simplifies an otherwise too complicated problem space, helping to clarify relationships by collecting sufficient data on specific cases (e.g., for a specific action).

\subsection{Neurobiological substrates}

Neurobiological analogies to the Q-Tree model parameters exist (e.g., Botvinick et al., 2008;

Daw, Niv, \& Dayan, 2005; Doya, 1999, 2002). For example, Doya $(1999,2002)$ proposed that 
the learning rate $\alpha$ is regulated by the neurotransmitter acetylcholine, and the exploring temperature $\tau$ is regulated by noradrenaline. In addition, the confusion effect $\kappa$ might be internally signaled by brain regions subserving response biases, such as the basal ganglia (Kandel et al., 2013). Finally, we propose that the sampling window $\omega$ reflects the rate at which implicit cognitive processing influences explicit processes, and more generally, that the overall coordination of these processes — our Q-Tree algorithm in general — provides a computational model of cognitive control, involving both implicit processing components, i.e., those conducted "in the background" (e.g., data history collection), and explicit ones (e.g., working memory and decision making on each trial) (e.g., Dijksterhuis et al., 2006; Miller \& Cohen, 2001). There is substantial evidence that prefontal cortex, especially 'granular' PFC, including both lateral PFC and frontal pole, subserves cognitive control, and plays a significant role in insightful problem solving (Bowden et al., 2005; Fuster, 2008; Gazzaniga et al., 2013; Kounios, et al., 2006; Miller \& Cohen, 2001; Passingham \& Wise, 2012; Striedter, 2005).

\subsection{Alternative models}

Regarding the cognitive models proposed by other authors for creative problem solving, and in particular, for incubation, restructuring, and insight, most are qualitative, which is indeed an important step in our understanding of the empirical phenomena (see van Steenburgh et al., 2012; Hélie and Sun, 2010). However, they then require extension to computational modeling for

clarification and detailed delineation of the relevant factors. Hélie and Sun (2010) have taken this next step in creative problem solving. They developed a model that focuses on two types of processing (explicit and implicit), each occurring within a separate module. The underlying structure within each module is a simple two-layer (i.e., input-output) connectionist network. In the explicit module, each node represents a particular concept (e.g., bartender, patron, glass of 
water, surprise), with the input nodes representing the main components of the problem and the output nodes potential solutions to the problem. The explicit module is considered rule-based and is constrained by pre-existing connections between input and output concepts (i.e., rules). For the implicit module, these same concepts are represented but rather than as one concept per node, each concept is represented across a series of nodes (and thus as a distributed representation, for both input and output layers). The connections between the input and output layers in the implicit module appear to be less constrained by prior knowledge, and provide a means for promoting more remote connections between problem components (input layer) and potentially correct answers (output layer). In any case, the interaction between the two modules leads to changes in the explicit input-output connections representing changes in the 'rules' (i.e., problem-solution mappings), producing the correct answer via insight (i.e., due to help from implicit processing). The model produces extended (considered incubation) and rapid (insight) periods of problem solving that match empirical date sets of human high-level cognition and insight problem solving. However, it is unclear how the model could obtain discontinuous learning curves like that found in reverse-reward problem solving that appear to reflect separable stages of explicit problem solving (stage 1), restructuring, incubation (stage 2), restructuring again, then insight (stage 3). To be sure, other straightforward reinforcement learning models with fixed state representation cannot account for these discontinuous learning curves.

In contrast, however, our model does account for these distinct stages, including multiple potential phases of problem restructuring. Thus, our model accounts for behavior in cases where there is no change in overt performance, but the individual nevertheless appears to be problem solving (implicitly). In addition, even though the three stages of problem solving exhibited by the rhesus monkeys may suggest three qualitatively different underlying processes, the 
discontinuous learning curves emerge from our single algorithm. As stated, the algorithm was initially developed as a straightforward means by which organisms can reduce complexity via a hierarchical scheme that clusters states with similar policies for attaining goals. Thus, our model proposes that complexity reduction and creativity are two sides of the same coin for higherfunctioning organisms, in which a cognitive control mechanism essentially toggles between the two general processes.

\subsection{Robot implementation}

Although computational modeling (with simulations) is a critical step forward in understanding high-level cognition, the approach nonetheless runs the risk of potential oversimplification of the real-world problem facing autonomous systems (such as using hand-coded inputs). Indeed, it has been forcefully argued that the understanding of cognitive processing is incomplete without considering its embodiment in a physical system (e.g., Pfeifer et al., 2007; Varela et al., 1991). Thus, not only must a cognitive ability such as creativity be understood within a broader context of a complete behavioral act or circuit within individual organisms (as argued above), it also must be embedded within an even broader context that includes the physical environment in which the organism is interacting. However, to understand the nature of this physical interaction, we need to consider what the cognitive ability was designed for — its adaptive function. In fact, a complete understanding of any biological trait of interest requires a larger evolutionary perspective that is well-captured by Tinbergen's four main categories of significant factors: (1) the trait's function (i.e., what it was designed for; called adaptation); (2) constraints due to its evolutionary history (such as the raw material available to mold into an adaptation; called phylogeny); (3) mechanism (i.e., what is normally studied, including all external and internal physical factors); and (4) ontogeny, i.e., development and learning processes (Tinbergen, 1963). 
This larger theoretical framework, then, motivates the second aim of our study: the testing and development of higher cognition in robots. Indeed, our physical experiment showed that with a vision-equipped robot the model builds the requisite representation and solves the same insight problem from camera percepts. This successful physical embodiment of the model spotlights factors such as the ease with which the system should be able to generalize from the specific visual inputs during initial learning to quantities in general. Moreover, it heightens the potential value of optimizing parameters, for example, to minimize errors and speed up learning (Passingham \& Wise, 2012; Pfeifer et al., 2007). Indeed, the robot experiment showed how a robot can learn the reverse-reward problem as effectively as or more effectively than a nonhuman primate, given an appropriate parameterization of the Q-Tree model (27 trials vs. a minimum of $\sim 400$ trials). Finally, the robot implementation has now laid the groundwork for further research development.

\subsection{Scaling to more sophisticated types of creative problem solving}

Although we concentrated on a relatively simple example of creative problem solving using nonhuman primates, it nevertheless consisted of key elements of creativity and insight: (1) apparent (selecting the better option) and nonapparent (selecting the worse option to receive the better one) solutions; as well as (2) behavioral evidence for reaching an impasse, incubation, restructuring, and insight. Nonetheless, the reverse-reward problem is an example of a simpler version of creativity, what has been termed opportunistic insight, in which the problem solver is primed for the answer, but they nevertheless experience it rather than come to it themselves (van Steenburgh et al., 2012).

The algorithm, however, readily extends to cases in which the problem solver must generate the solution internally, which we have done with the classic 9-dot insight problem with humans 
(Cheng et al., 2013; van Steenburgh et al., 2012). The problem requires the participant to connect all nine dots by drawing only four lines without lifting the pen/pencil. The problem is very difficult for humans because we continue to attempt the apparent, efficient solution in which lines are drawn that begin and end at dots; yet the nonapparent solution requires the problem solver to draw lines that extend beyond the dots. In Cheng et al. (2013), we modeled the solution as a nonapparent action being added to the action set.

To extend the algorithm even further, future work will need to continue development of the (cognitive-control) mechanism for more sophisticated coordination of apparent versus nonapparent problem components (e.g., states, stimuli, actions). The algorithm would be expected to place a higher priority on representations and policies that have been previously successful. That is, while in this paper we referred to joinability and separability of the state space as giving rise to incubation dynamics and restructuring, there is evidence that in both humans and nonhuman animals that prior representations (via instincts or previously learned) are retained along with the new representation (Kandel et al., 2013). Thus, after restructuring, there exist distinct representations corresponding to different policies or "rules" governing action selection. In subsequent work, we investigate the use of Q-tree learning for building multiple policies and associated representations, incorporating mathematical constructs that provide evidence that an existing policy cannot solve a given problem, and a mechanism to either find the appropriate problem representation and policy (i.e., rule) in memory or build a new one. These constructs provide a more detailed computational account of cognitive control and working memory in the brain, and also speak to the nature of state-action transitions, e.g., whether they obey the Markov property or not. We can readily build on the Q-Tree algorithm to incorporate these concepts, thus establishing a single computational mechanism for policy 
selection and for building multiple representations for solving more complex problems creatively.

\section{Conclusions}

The proposed model using reinforcement learning and hierarchical state representation with a decision tree structure underscores the importance of generating appropriate state representations for problem solving to avoid impasses (i.e., structural credit-assignment problems) that occur due to incorrect internal beliefs of the problem solver's environment. Experiments with rhesus monkeys show that primates, or at least some catarrhine primates (Old World monkeys, apes, and humans), can resolve these problem-solving impasses with a long enough incubation period, and can transfer the learned knowledge to a new but similar problem. The Q-Tree learning model can produce the three stage learning curve observed with the monkeys, which a traditional reinforcement-learning model with fixed state representation cannot explain. Simulation of transfer test performance explains both how successful transfer may occur and why some novel quantity combinations are more difficult to generalize to than others. Extension of the model to a robot produces a similar three-stage learning curve, but with parameters tuned to enable more rapid progression through the stages, with the robot learning the new representation in 40 trials (vs. 400 trials for the best-performing rhesus monkey). Our model provides insight into how the creative brain may address the dual challenges of complex environments — finding unprecedented opportunity hidden within potentially intractable complexity — and suggests that both challenges may be met by a single underlying computational mechanism.

\section{Acknowledgment}

This work was sponsored by the Office of Naval Research under Multi-University Research Initiative (MURI) Grant No. N00014-08-1-0693. 


\section{References}

Banerjee, K., Chabris, C.F., Johnson, V.E., Lee, J.J., Tsao, F., Hauser, M.D. (2009) 'General Intelligence in Another Primate: Individual Differences across Cognitive Task Performance in a New World Monkey (Saguinus oedipus).” Plos One, 4(6).

Botvinick, M., Niv, Y., and Barto, A.C., "Hierarchically organized behavior and its neural foundations: a reinforcement learning perspective," Cognition, 1-19.

Bowden, E. M., Jung-Beeman, M., Fleck, J., \& Kounios, J. (2005). New approaches to demystifying insight. Trends in Cognitive Sciences, 9(7), 322-328.

Cheng, Z., Ray, L. E., Nguyen, H. T., and Kralik, J. D. (2013). Modeling the Aha! moment: A computational mechanism for structuring and incubation in creative problem solving. Proceedings of the International Association of Science and Technology for Development (IASTED) International Conference on Intelligent Systems and Control (ISC).

Crites, R.H., and Barto, A.G. (1995) "An actor/critic algorithm that is equivalent to Q-learning," Advances in Neural Information Processing Systems, 7, 401-408.

Dehaene, S., Dehaene-Lambertz, G., and Cohen, L. (1998) "Abstract representation of numbers in the animal and human brain," Trends in Neuroscience, 21, 355-361.

Dijksterhuis, A., Bos, M. W., Nordgren, L. F., \& van Baaren, R. B. (2006). On making the right choice: The deliberation-without-attention effect. Science, 311(5763), 1005-1007

Doya, K., (1999) "What are the computations in the cerebellum, the basal ganglia, and the cerebral cortex," Neural Networks, 12, 234-240.

Doya, K. (2002) "Metalearning and neuromodulation," Neural Networks, 495-506, 2002.

Feigenson, L., Dehaene, S., and Spelke, E. (2004) "Core systems of number," Trends in Cognitive Science, 8, 307-314.

Fuster, J. M. (2008). The prefrontal cortex. London: Academic Press.

Gazzaniga, M. S., Ivry, R. B., and Mangun, G. R. (2013). Cognitive Neuroscience. New York: W. W. Norton \& Company.

Hélie, S. and Sun, R. (2010) "Incubation, Insight, and Creative Problem Solving: A Unified Theory and a Connectionist Model," Psychological Review, 117(3), 994-1024.

Holyoak, K. J. and Morrison, R. G. (2012). The Oxford Handbook of Thinking and Reasoning. Oxford: Oxford University Press.

Kahneman, D. (2011). Thinking, fast and slow. New York: Farrar, Straus, and Giroux.

Kahneman, D., Slovic, P., \& Tversky, A. (Eds.). (1982). Judgement under uncertainty: Heuristics and biases. Cambridge: Cambridge University Press.

Kandel, E. R., Schwartz, J. H., Jessell, T. M., Siegelbaum, S. A., and Hudspeth, A. J. (2013). Principles of Neural Science. New York: McGraw-Hill.

Kralik, J.D., (2005) "Inhibitory control and response selection in problem solving: How cottontop tamarins (Saguinus oedipus) overcome a bias for selecting the larger quantity of food," Journal of Comparative Psychology, 119(1), 78-89.

Kralik, J.D., (2011) "Rhesus Macaques (Macaca mulatta) spontaneously generalize to novel quantities in a reverse-reward contingency task," Journal of Comparative Psychology, 255262.

Kralik, J. D. , Hauser, M. D., and Zimlicki, R., (2002) "The relationship between problem solving and inhibitory control: Cotton-top tamarine (Saguinus oedipus) performance on a reverse contingency task," Journal of Comparative Psychology, 116(1), 39-50.

Kohler, W. (1925). The mentality of apes. London: Kegan Paul, Trench, Trubner. 
Kounios, John, Frymiare, Jennifer L., Bowden, Edward M., Fleck, Jessica I., Subramaniam, Karuna, Parrish, Todd B., et al. (2006). The prepared mind - Neural activity prior to problem presentation predicts subsequent solution by sudden insight. Psychological Science, 17(10), 882-890.

Mao, T., Cheng, Z. and Ray, L. (2012) 'Q-Tree: Automatic Construction of Hierarchical State Representations for Reinforcement Learning," 2012 International Conference on Intelligent Robotics and Applications.

Miller, E. K., \& Cohen, J. D. (2001). An integrative theory of prefrontal cortex function. Annual Review of Neuroscience, 24, 167-202.

Murray, E.,A. Kralik, J. D., and Wise, S. P., (2005) "Learning to inhibit prepotent responses: successful performance by rhesus macaques, Macaca mulatta, on the reversed-contingency task," Animal Behavior, 69, 991-998.

Passingham, R. E., \& Wise, S. P. (2012). The neurobiology of the prefrontal cortex: Anatomy, evolution, and the origin of insight. Oxford UK: Oxford University Press.

Pfeifer, R., Lungarella, M., Iida, F. (2007). Self-organization, embodiment, and biologically inspired robotics. Science, 318, 1088-1093.

Pyeatt, L. and Howe, A. (2001) "Decision tree function approximation in reinforcement learning," in Proc. 3rd Int'l Symposium on Adaptive Systems: Evolutionary Computation and Probablistic Graphical Models.

Russell, S., \& Norvig, P. (2010). Artificial Intelligence: A Modern Approach. Upper Saddle River, NJ: Prentice Hall.

Shifferman, E. M. (2009) "Its own reward: lessons to be drawn from the reversed-reward contingency paradigm," Animal Cognition, 12(4), 547-558.

Smith, S. M., \& Ward, T. B. (2012). Cognition and the creation of ideas. In K. J. Holyoak \& R. G. Morrison (Eds.), The Oxford Handbook of Thinking and Reasoning. Oxford: Oxford University Press.

Striedter, G. F. (2005). Principles of brain evolution. Sunderland: Sinauer Associates.

Sun, X., Mao, T., Ray, L., Shi, D., \& Kralik, J. (2011). Hierarchical state-abstracted and socially augmented Q-Learning for reducing complexity in agent-based learning. Journal of Control Theory and Applications, 9(3)

Sutton, R. S. and Barto, A. G. (1998) Reinforcement Learning: An Introduction, Cambridge, MA, USA: MIT Press.

Taylor, M. E. and Stone, P., (2009) "Transfer learning for reinforcement learning domain: a survey," Journal of Machine Learning Research, 10, 1633-1685.

Tinbergen, N. (1963). On Aims and Methods of Ethology, Zeitschrift für Tierpsychologie, 20, $410-433$.

Uther, W., and Veloso, M. (1998) "Tree-based discretization for continuous state space reinforcement learning," in Proc. of 16th National Conf. on Artificial Intelligence (AAAI), Madision, WI, USA.

van Steenburgh, J. J., Fleck, J. I. , Beeman, M., and Kounios, J. (2012) "Insight," in The Oxford Handbook of Thinking and Reasoning, Oxford, Oxford University Press.

Varela, F. J., Thompson, E., and Rosch, E. (1991) The Embodied Mind, Cambridge, MA, USA: MIT Press. 